\title{
Development and significance of SCAR marker QG12-5 for Canarium album (Lour.) Raeusch by molecular cloning from improved RAPD amplification
}

J.L. Cheng ${ }^{1 *}$, Z.C. Yin ${ }^{2,3 *}$ Z Q. Mei ${ }^{1}$ C.L. Wei ${ }^{1,4}$, H.C. Chen ${ }^{5}$, X.S. W ${ }^{3}$ and J.J. Fu $\mathbf{u}^{1,4,6}$

${ }^{1}$ Research Center for Preclinical Medicine, Southwest Medical University, Luzhou, Sichuan, China

${ }^{2}$ Key Laboratory of Genetics and Birth Health of Hunan Province, Family Planning Institute of Hunan Province, Changsha, Hunan, China ${ }^{3}$ Key Lab of MOE for Development Biology and Protein Chemistry, Center for Heart Development, College of Life Sciences, Hunan Normal University, Changsha, Hunan, China ${ }^{4}$ State Key Laboratory of Quality Research in Chinese Medicine, Macau University of Science and Technology, Macau, China ${ }^{5}$ Department of Biochemistry, School of Life Sciences \& the State Key Laboratory of Medical Genetics, Central South University, Changsha, Hunan, China ${ }^{6}$ Judicial Authentication Center, Southwest Medical University, Luzhou, Sichuan, China

*These authors contributed equally to this study.

Corresponding authors: X.S. Wu / J.J. Fu

E-mail: xiushanwu2003@aliyun.com / fujunjiang@hotmail.com

Genet. Mol. Res. 15 (3): gmr.15038347

Received December 17, 2015

Accepted February 11, 2016

Published August 26, 2016

DOI http://dx.doi.org/10.4238/gmr.15038347

Copyright (C 2016 The Authors. This is an open-access article distributed under the terms of the Creative Commons Attribution ShareAlike (CC BY-SA) 4.0 License.

ABSTRACT. Sequence-characterized amplified region (SCAR) is a valuable molecular marker for the genetic identification of any species. 
This marker is mainly derived from molecular cloning of random amplified polymorphic DNA (RAPD). We have previously reported the use of an improved RAPD technique for the genetic characterization of different samples of Canarium album (Lour.) Raeusch (C. album). In this study, DNA fragments were amplified using improved RAPD amplified from different samples of C. album. The amplified DNA fragment was excised, purified from an agarose gel and cloned into a pGM-T vector; subsequently, a positive clone, called QG12-5 was identified by PCR amplification and enzymatic digestion and sequenced by Sanger di-deoxy sequencing method. This clone was revealed consisting of 510 nucleotides of C. album. The SCAR marker QG12-5 was developed using specifically designed PCR primers and optimized PCR conditions. This SCAR marker expressed seven continuous "TATG" $[($ TATG $) n]$ tandem repeats, which was found to characterize C. album. Subsequently, this novel SCAR marker was deposited in GenBank with accession No. KT359568. Therefore, we successfully developed a C. album-specific SCAR marker for the identification and authentication of different $C$. album species in this study.

Key words: Canarium album; Molecular cloning; Genetic characterization; Random amplified polymorphic DNA; Sequence-characterized amplified region

\section{INTRODUCTION}

The Chinese olive tree Canarium album (Lour.) Raeusch, called Qingquo (Qīng guǒ) in Chinese, is an evergreen native plant seen in southeastern China, particularly in the Guangdong, Hainan, Sichuan, Guangxi, and Fujian provinces. So far, C. album has also been introduced to other Asian tropical and semi-tropical regions, including Vietnam, Japan, and Malaysia (Mei et al., 2014). The C. album fruit is a drupe, and is similar to the Mediterranean olive (Olean europaea L.) in terms of the type and anatomy. The fruits, nuts, seeds, and roots of $C$. album have been historically used as traditional Chinese medicines (TCM) for the treatment of swollen and sore throat, excessive thirst, hematemesis due to cough, lacillary dysentery, epilepsy, puffer poisoning, and alcoholism (Wang et al., 2010; Mei et al., 2014).

Studies have indicated that compounds from C. album, including triterpenes, brevifolin, hyperin, ellagic acid, and 3,3'-di-O-methylellagic acid, also exhibit hepatoprotective activities. Moreover, they are believed to exhibit antioxidant effects in vitro and in vivo (Tamai et al., 1989; Ito et al., 1990; Wang et al., 2010). The dried fruits are also reported to have anti-viral, anti-bacterial, anti-inflammatory, and detoxifying effects (Zhang and Lin, 2008). The Hejiang County of Luzhou City in the Sichuan Province of China, which lies upstream of the Yangtze River, is the largest producer of C. album in Sichuan. The medicinal activities of C. album products obtained from different cultivars and/or localities are known to vary. Therefore, it is important to genetically characterize and identify "good" C. album species and/or cultivars.

Random amplified polymorphic DNA (RAPD), inter-simple sequence repeat (ISSR), simple sequence repeat (SSR), and amplified fragment length polymorphism or restriction fragment length polymorphism analyses are the major molecular marker technologies that

Genetics and Molecular Research 15 (3): gmr.15038347 
are currently being used for the genetic characterization and identification of an organism (Williams et al., 1990; Agarwal et al., 2008; Noormohammadi et al., 2013; Mei et al., 2014; Fu et al., 2000, 2013, 2015; Long et al., 2015; Zhan et al., 2015). The sequence characterized amplified region (SCAR) marker is another molecular marker that is more stable and is generally derived from RAPD, ISSR, or other markers (Dnyaneshwar et al., 2006; Su et al., 2007; Kumla et al., 2012; Fu et al., 2013; Rajesh et al., 2013; Mei et al., 2015; Zhang et al., 2015). Combining SCAR with RAPD simplifies the molecular analysis procedure, as the PCR primers used to develop SCAR markers are designed from the sequence of the RAPD amplicon (Kumla et al., 2012; Rajesh et al., 2013; Dutta et al., 2014; Fu et al., 2015).

We have previously developed an improved RAPD technique for the genetic characterization of different samples for C. album (Mei et al., 2014). In this study, the SCAR marker was developed by cloning RAPD-DNA fragments obtained from various $C$. album. We discovered that this marker was specific for C. album.

\section{MATERIAL AND METHODS}

\section{C. album sample collection and DNA extraction}

DNA was extracted from different samples of C. album (Mei et al., 2014) and other medicinal plant samples using a standard protocol (Fu, 2012; Fu et al., 2000, 2013, 2015; Khan et al., 2015). DNA samples were then diluted to a final concentration of $10 \mathrm{ng} / \mathrm{mL}$ and stored at $-20^{\circ} \mathrm{C}$ until further use, as described in a previous study (Fu et al., 2015).

\section{Amplification and recovery of improved RAPD fragments}

Improved RAPD fragments from 8 C. album samples were amplified by polymerase chain reaction (PCR), using the random primer SBC-Q12. The protocol used for amplification and recovery of improved RAPD fragments was as described previously (Fu et al., 2015). Briefly, the 15-mL PCR mixture, comprising $7.5 \mathrm{~mL} 2 \mathrm{X}$ Taq PCR MasterMix (Tiangen Biotech, Beijing, China), $1.5 \mathrm{~mL} 2.5 \mathrm{mM}$ primer pairs, $1.5 \mathrm{~mL}$ genomic DNA, and $\mathrm{ddH}_{2} \mathrm{O}$, was amplified on a Veriti ${ }^{\circledR}$ 96-Well thermal cycler (Applied Biosystems, Foster City, CA, USA), using the following reaction protocol: initial denaturation at $95^{\circ} \mathrm{C}$ for $90 \mathrm{~s} ; 40$ cycles of denaturation at $94^{\circ} \mathrm{C}$ for $40 \mathrm{~s}$, annealing at $36^{\circ} \mathrm{C}$ for $60 \mathrm{~s}$, and extension at $72^{\circ} \mathrm{C}$ for $90 \mathrm{~s}$, with the annealing to extension temperature increasing at a rate of $0.125^{\circ} \mathrm{C} / \mathrm{s}(5 \%$ ramp rate $)$; and a final extension at $72^{\circ} \mathrm{C}$ for $5 \mathrm{~min}$. PCR products were separated on a $1.5 \%$ agarose gel for $40 \mathrm{~min}$. The bright bands were excised from the agarose gel, and purified using the TIANgel Mini Purification Kit (DP209; Tiangen Biotech, Beijing, China) according to the manufacturer protocols. Thus, purified DNA was obtained from the RAPD fragments.

\section{Cloning, screening, and sequencing of RAPD DNA fragments}

The purified RAPD fragments and pGM-T vector (No. VT202; Tiangen) were ligated at an approximate molar ratio of 3:1, and subsequently transformed into DH5 $\alpha$ Escherichia coli competent cells. Recombinant clones were selected on Luria Bertani agar plates containing ampicillin $(100 \mu \mathrm{g} / \mathrm{mL}$, final concentration), 5-bromo-4-chloro-3-indoyl$\beta$-D-galactopyranoside (40 mg), and isopropyl $\beta$-D-1-thiogalactopyranoside $(160 \mu \mathrm{g})$. White

Genetics and Molecular Research 15 (3): gmr.15038347 
colonies were screened out by blue/white screening. The presence of the correct insert was verified by PCR using the T7/SP6 primer pairs, and subsequently by EcoRI digestion (Fu et al., 2015). The cloned DNA fragments were then sequenced using the Sanger di-deoxy sequencing method.

\section{Homology analysis and SCAR primer design}

Homology matches of the sequenced DNA in different species were identified in the GenBank database and analyzed online using Basic Local Alignment Search Tool (BLAST; http://www.ncbi.nlm.nih.gov/BLAST/). The nucleotide sequence of the cloned RAPD fragment was used to design pairs of SCAR primers using the Primer 3 program (http:// bioinfo.ut.ee/primer3-0.4.0/primer3/). Primer pair sequences are listed in Table 1.

Table 1. Primer sequences for rapid amplified polymorphic DNA (RAPD) and sequence-characterized amplified region (SCAR) amplification.

\begin{tabular}{l|l|c|c}
\hline Primer Name & Sequence 5' $\rightarrow 3^{\prime}$ & Size $(\mathrm{bp})$ & Tm $\left({ }^{\circ} \mathrm{C}\right)$ \\
\hline SBS-Q12 & AGTAGGGCAC & & \\
\hline QG12-5L-full & AGTCCTATATCTTGCTATCAATCTCT & 510 & \\
\hline QG12-5R-full & TCACATGAATGTTAGTCGAGTTTTC & & \\
\hline QG12-5L & TCCGATGGTCGCATCTATTT & 292 & \\
\hline QG12-5R & GGTCCATTCAAGAGGTCCAA & & 60 \\
\hline
\end{tabular}

$\mathrm{Tm}=$ melting temperature.

\section{Development of SCAR markers and SCAR analysis}

SCAR markers were developed by PCR amplification using a $10-\mathrm{mL}$ system, comprising $5 \mathrm{~mL} 2 \mathrm{X}$ Taq PCR MasterMix (Tiangen Biotech, Beijing, China), $1 \mathrm{~mL}$ of each 2.5 mM SCAR primer (QG12-5L-full and QG12-5R-full, or QG12-5L and QG12-5R), $1 \mathrm{~mL}$ (10

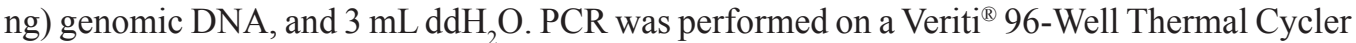
(Applied Biosystems) using the following reaction conditions: initial pre-denaturation at $95^{\circ} \mathrm{C}$ for $90 \mathrm{~s} ; 35$ cycles of denaturation at $94^{\circ} \mathrm{C}$ for $40 \mathrm{~s}$, annealing at $60^{\circ} \mathrm{C}$ for $30 \mathrm{~s}$, and extension at $72^{\circ} \mathrm{C}$ for $40 \mathrm{~s}$; and a final extension at $72^{\circ} \mathrm{C}$ for $5 \mathrm{~min}$. SCAR primer sequences, lengths of the amplified fragments, and PCR conditions are listed in Table 1. Differences between the varieties of C. album and other species of medicinal plants were identified by SCAR analysis, using 18 template DNA samples, including 8 samples of C. album, 2 of Dimocarpus longan collected from Fujian and Hainan, 1 of Lonicera japonica collected from Hunan, 1 of Gardenia jasminoides, 1 sample of Litchi chinensis from Guangdong, 1 of Dimocarpus confinis from Guangxi, 1 of Gastrodia elata collected from Liangshan City in Sichuan Province, 1 sample of Penthorum chinense collected from Gulin County in Sichuan Province, 1 sample of Viola philippica, and 1 sample of Angelica sinensis from Sichuan Province, and one empty control without DNA. Six new C. album samples from Meizhou and Maoming of Guangdong $(\mathrm{N}=$ 2), Fuzhou and Zhangzhou of Fujian ( $\mathrm{N}=2)$, Guangxi $(\mathrm{N}=1)$, and Xizang $(\mathrm{N}=1)$ were also collected. The amplified PCR products were separated by electrophoresis on a $1.5 \%$ agarose gel in $1 \mathrm{X}$ TAE buffer, at $120 \mathrm{~V}$ for $40 \mathrm{~min}$, or on an $8 \%$ PAGE gel at $120 \mathrm{~V}$ for $30 \mathrm{~min}$. The gels were visualized by $0.5 \mathrm{mg} / \mathrm{mL}$ ethidium bromide staining or silver staining (Fu, 2012), and the images were documented using ChemiDoc XRS (Bio-Rad, Hercules, CA, USA).

Genetics and Molecular Research 15 (3): gmr.15038347 


\section{RESULTS}

\section{Recovery of DNA fragments from improved RAPD amplification}

The primer SBC-Q12 (Table 1) was used to improve RAPD amplification from $C$. album DNA samples. The results of this amplification are shown in Figure 1A; the blue arrow indicates the band cut out from the agarose gel. DNA extracted from the agarose gel was purified using the TIANgel Mini Purification Kit and eluted with $20 \mathrm{~mL} \mathrm{ddH}_{2} \mathrm{O}$. DNA quality and the quantity required for ligation were determined by adding $2 \mathrm{~mL}$ purified PCR product and $0.5 \mathrm{~mL} \mathrm{T-vector} \mathrm{to} \mathrm{each} \mathrm{well} \mathrm{of} \mathrm{an} \mathrm{agarose} \mathrm{gel} \mathrm{(data} \mathrm{not} \mathrm{shown).}$

A

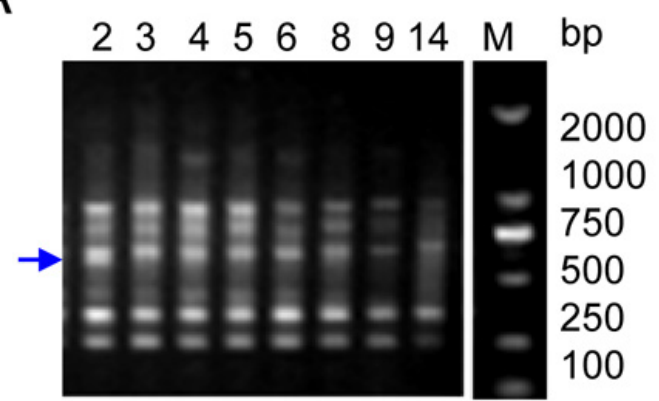

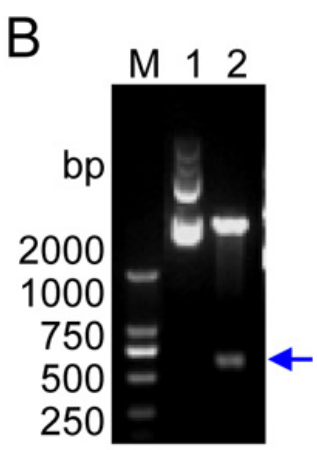

Figure 1. Improved RAPD amplification and molecular cloning. A. Improved RAPD amplification from genomic DNA extracted from Canarium album (Lour.) Raeusch. Lanes 2-6, 8, 9, and 14 indicate the different samples of $C$. album (Mei et al., 2014). The blue arrow indicates the selected and cut band. B. Identification of positive clones by plasmid DNA digestion. Lanes 1 and 2 indicate the EcoRI-digested and -undigested plasmid DNA of clone QG125. The blue arrow indicates the expected insert of the RAPD DNA fragment. Lane $M$ indicates the standard DNA molecular weight marker DL2000.

\section{Cloning of DNA fragments}

An appropriate amount of PCR product (selected based on the DNA quality) was ligated with the T-vector (3:1 molar ratio) and screened. Positive clones were identified by PCR amplification using the SP6/T7 primers (data not shown). Plasmids were extracted from the positive clones and cut using the EcoRI restriction enzyme (Figure 1B). Clone QG12-5, extracted from lane 2 of Figure 1B, which showed a 550-base pair (bp) DNA fragment, was sequenced.

\section{Sequencing and characterization of $C$. album species-specific RAPD fragments}

The cloned RAPD fragment QG12-5 was sequenced and the results revealed that this clone consisted of 510 nucleotides. The sequence was deposited in GenBank with accession No. KT359568 (Figure 2). BLAST searches of homologous nucleotide sequences in the GenBank database revealed that the cloned DNA from C. album did not share any identity with those from other species (data not shown). Careful analysis of QG12-5 showed the presence of seven consecutive "TATG" [(TATG)n] tandem repeats. 


\section{AGTCCTATATCTTGCTATCAATCTCTCTTCAGGGCTCTGAGAATCCGATGGTCGCATCTATTTATATATATAGATATATA \\ 81 TGTATGTATGTATGTATGTATGTATGTATTGATGTAGAATAAATCTACGTAATTTAACACTCAATTAATTCAACTCAAAT \\ 161 CTCATTCAATCATCAAAAAACCGTCTTACAAAATAATAACCTAGCCTTTATATAGGCTCAAAACCCTAAACAAATATTAA \\ 241 ATGCCATAATTATCCTTAAAGTCTAAATTAACAATAGTAGATAATTAACATATGGGTTGGATTCTGGATTTGGGCTTGGA \\ 321 CCTCTTGAATGGTCCTCCATCATTCCCCCCGGGTTGAAAAGAACTCGACCCAGGAGAGTTAGTTGTAGTCAATCCTCCAT \\ 401 CTGAAGAAGCTGTACCTGTAGAAGCATGAGCAGACAAAATGGGAGGCTTACAAGTACCTCGAAAAGGTGTCAGATCCTCC \\ 481 ACATTGAAAACTCGACTAACATTCATGTGA}

Figure 2. Information regarding the sequenced clone. Sanger-sequencing of the clone QG12-5 produced a 510-bp fragment, which was deposited into GenBank with the accession No. KT359568.

\section{Development of $C$. album species-specific SCAR marker}

Stable diagnostic C. album species-specific SCAR markers were developed from RAPD markers using PCR primers (QG12-5L-full and QG12-5R-full) (Table 1) designed and synthesized based on the cloned sequences. The designed SCAR primer pairs were then used to amplify genomic DNA from the 18 collected DNA samples to test the species-specificity of amplification. The results of this analysis are shown in Figure 3A: the size of the SCAR maker QG12-5 was as expected in all eight C. album samples (Figure 3A); none of the other tested species showed any amplification. The amplification of 6 more C. album samples (Figure 3B) provided the same results. These results indicate the species-specificity of this SCAR marker. The lack of this specific amplicon in other species indicates the efficacy of this marker in distinguishing C. album.

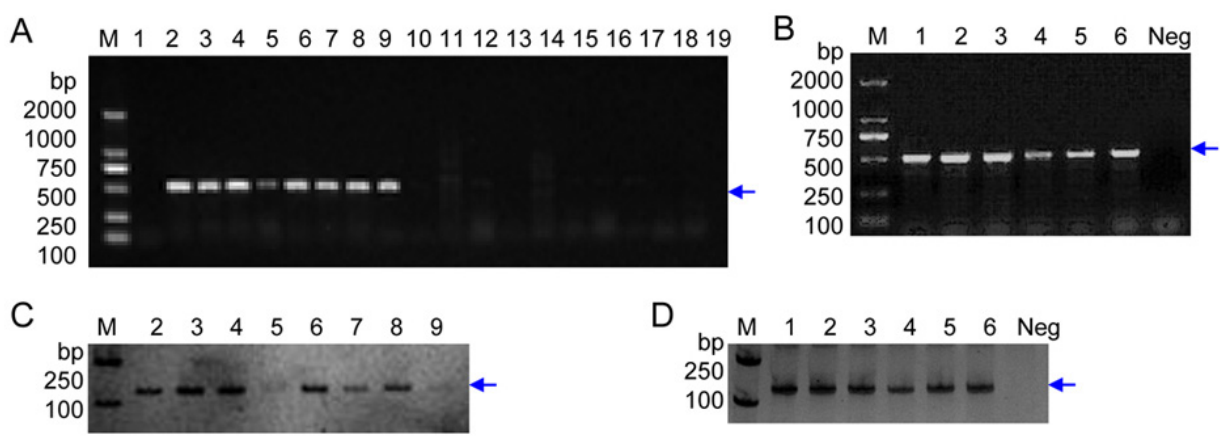

Figure 3. Development and verification of RAPD-SCAR marker QG12-5. A. SCAR marker QG12-5 was amplified in Canarium album from Luzhou and other species. Lanes 2-9 indicate the different samples of C. album (Lour.) Raeusch (Mei et al., 2014); lanes 10 and 11 indicate the amplification results of Dimocarpus longan; and lanes 12-19 indicate the separated amplified fragments of Lonicera japonica, Gardenia jasminoides, Litchi chinensis, Dimocarpus confinis, Gastrodia elata, Penthorum chinense, Viola philippica, and Angelica sinensis, respectively. Lane 1 is a control lane without DNA. B. SCAR marker QG12-5 was amplified in C. album from different localities. Lanes 1-6 indicate the amplified DNA fragments from two samples obtained from Meizhou and Maoming of the Guangdong Province, Fuzhou and Zhangzhou of the Fujian Province, Guangxi, and Xizang, respectively, while lane 7 was the control sample containing no DNA. C. D. SCAR marker QG12-5 was amplified using the primers QG12-5L and QG12-5R, and analyzed by silver staining. C. The lanes 2-9 indicate the amplification results of various C. album species, as detailed in A. D. Lanes 1-7 indicate the amplification results of various C. album species, as detailed in in C. The blue arrow indicates the expected size of PCR products. Lane $M$ indicates the DNA molecular weight marker DL2000 or DL600, with their indicated sizes.

Genetics and Molecular Research 15 (3): gmr.15038347 


\section{Polymorphic analysis for SCAR marker QG12-5 in C. album samples}

The sequencing results revealed the presence of seven continuous "TATG" [(TATG) n] tandem repeats in the clone QG12-5; therefore, the DNA of fourteen C. album species or cultivars were amplified by PCR, using the primers QG12-5L and QG12-5R, which amplified shorter amplicons for polymorphic study. The amplified fragments were separated on an $8 \%$ PAGE gel and analyzed by silver staining. The analyzed samples showed no polymorphic bands (Figure 3C and D).

\section{DISCUSSION}

Molecular markers, particularly RAPD markers, and improved RAPD analysis are common and efficient tools for the molecular analysis or genetic characterization of different species. RAPD and other markers have been widely used to characterize several fruity and medicinal trees, including C. album. Yang et al. (2011) and Chen et al. (2011) genetically characterized C. album from the Fujian and the eastern Guangdong provinces, respectively, using the ISSR technology. Martins-Lopes et al. (2008) evaluated the efficiency of RAPD, ISSR, and SSR molecular markers in the varietal identification of olive oil. In a previous study, we used an improved RAPD analysis method to characterize C. album samples from the Sichuan province along the Yangtze River (Mei et al., 2014). Zhan et al. (2015), on the other hand, characterized olive trees from Xichang, in the Sichuan Province of China, using ISSR and SSR. However, no previous studies have used SCAR markers to identify different $C$. album species. In this study, we developed the SCAR molecular marker QG12-5 to genetically identify the C. album genus, based on the previously reported improved RAPD characterization.

The 510-nucleotide SCAR marker QG12-5, which was developed to identify or authenticate C. album, was deposited in GenBank with accession No. KT359568. BLAST searches of the nucleotide sequences in GenBank revealed no matches to these known sequences; therefore, we concluded that this novel marker could be used to differentiate $C$. album species from others. Interestingly, careful analysis of the QG12-5 cloned sequence revealed the presence of seven continuous "TATG" [(TATG)n] tandem repeats, indicating its potential for cultivar identification, despite the lack of polymorphic bands in our analyzed samples. Several samples must be collected and analyzed in the future to validate our results. High genetic variability among species enabled the generation of a C. album-specific SCAR marker. Therefore, this molecular marker may play a major role in ecological preservation, molecular authentication, and genetic characterization of this edible and medicinal plant.

\section{Conflicts of interest}

The authors declare no conflict of interest.

\section{ACKNOWLEDGMENTS}

Research supported partly by the Science and Technology Innovation Team of Colleges and Universities in Sichuan Province (\#13TD0032), the Applied Basic Research Program of Science and Technology Department of Sichuan Province (\#14JC0797 and \#2015JY0038), and the Southwest Medical University Foundation (\#2014QN091).

Genetics and Molecular Research 15 (3): gmr.15038347 


\section{REFERENCES}

Agarwal M, Shrivastava N and Padh H (2008). Advances in molecular marker techniques and their applications in plant sciences. Plant Cell Rep. 27: 617-631.http://dx.doi.org/10.1007/s00299-008-0507-z

Chen MX, Zhang QJ, Chen QX and Lin YF (2011). ISSR analysis of main cultivated and fresh-eating Canarium album (Lour.) Raeusch in Fuzhou city. Modern Agric. Sci. Tech. 21: 129-130.

Dnyaneshwar W, Preeti C, Kalpana J and Bhushan P (2006). Development and application of RAPD-SCAR marker for identification of Phyllanthus emblica LINN. Biol. Pharm. Bull. 29: 2313-2316.http://dx.doi.org/10.1248/bpb.29.2313

Dutta S, Biswas S, Mukherjee K, Chakrabarty U, et al. (2014). Identification of RAPD-SCAR marker linked to white spot syndrome virus resistance in populations of giant black tiger shrimp, Penaeus monodon Fabricius. J. Fish Dis. 37: 471-480. http://dx.doi.org/10.1111/jfd.12128

Fu JJ (2012). Short protocols in medical molecular biology. China Medical Science Press, Beijing.

Fu JJ, Li LY, Xu X, Wang Z, et al. (2000). An improved method for increasing the efficiency of the technique of Random Amplified Polymorphic DNA (RAPD). Hereditas 22: 251-252.

Fu J, Yang L, Khan MA and Mei Z (2013). Genetic characterization and authentication of Lonicera japonica Thunb. by using improved RAPD analysis. Mol. Biol. Rep. 40: 5993-5999. http://dx.doi.org/10.1007/s11033-013-2703-3

Fu JJ, Mei ZQ, Tania M, Yang LQ, et al. (2015). Development of RAPD-SCAR markers for different Ganoderma species authentication by improved RAPD amplification and molecular cloning. Genet. Mol. Res. 14: 5667-5676. http:// dx.doi.org/10.4238/2015.May.25.19

Ito M, Shimura H, Watanabe N, Tamai M, et al. (1990). Hepatoprotective compounds from Canarium album and Euphorbia nematocypha. Chem. Pharm. Bull. (Tokyo) 38: 2201-2203.http://dx.doi.org/10.1248/cpb.38.2201

Khan MA, Tania M, Wei C, Mei Z, et al. (2015). Thymoquinone inhibits cancer metastasis by downregulating TWIST1 expression to reduce epithelial to mesenchymal transition. Oncotarget 6: 19580-19591. http://dx.doi.org/10.18632/ oncotarget. 3973

Kumla S, Doolgindachbaporn S, Sudmoon R and Sattayasai N (2012). Genetic variation, population structure and identification of yellow catfish, Mystus nemurus (C\&V) in Thailand using RAPD, ISSR and SCAR marker. Mol. Biol. Rep. 39: 5201-5210.http://dx.doi.org/10.1007/s11033-011-1317-x

Long Y, Cheng J, Mei Z, Zhao L, et al. (2015). Genetic analysis of litchi (Litchi chinensis Sonn.) in southern China by improved random amplified polymorphic DNA (RAPD) and inter-simple sequence repeat (ISSR). Mol. Biol. Rep. 42: 159-166. http://dx.doi.org/10.1007/s11033-014-3755-8

Martins-Lopes P, Gomes S, Santos E and Guedes-Pinto H (2008). DNA markers for Portuguese olive oil fingerprinting. $J$. Agric. Food Chem. 56: 11786-11791.http://dx.doi.org/10.1021/jf801146z

Mei Z, Yang L, Zhang T, Yu H, et al. (2014). Improved RAPD analysis of Canarium album (Lour.) Raeusch from Sichuan province along Yangtze River in China. Annu. Res. Rev. Biol. 4: 51-60. http://dx.doi.org/10.9734/ARRB/2014/5015

Mei Z, Zhou B, Wei C, Cheng J, et al. (2015). Genetic authentication of Gardenia jasminoides Ellis var. grandiflora Nakai by improved RAPD-derived DNA markers. Molecules 20: 20219-20229. http://dx.doi.org/10.3390/ $\underline{\text { molecules } 201119687}$

Noormohammadi Z, Hasheminejad-Ahangarani Farahani Y, Sheidai M, Ghasemzadeh-Baraki S, et al. (2013). Genetic diversity analysis in Opal cotton hybrids based on SSR, ISSR, and RAPD markers. Genet. Mol. Res. 12: 256-269. http://dx.doi.org/10.4238/2013.January.30.12

Rajesh MK, Jerard BA, Preethi P, Thomas RJ, et al. (2013). Development of a RAPD-derived SCAR marker associated with tall-type palm trait in coconut. Sci. Hortic. (Amsterdam) 150: 312-316. http://dx.doi.org/10.1016/j. scienta.2012.11.023

Su H, Wang L, Ge Y, Feng E, et al. (2007). Development of strain-specific SCAR markers for authentication of Ganoderma lucidum. World J. Microbiol. Biotechnol. 24: 1223-1226. http://dx.doi.org/10.1007/s11274-007-9579-0

Tamai M, Watanabe N, Someya M, Kondoh H, et al. (1989). New hepatoprotective triterpenes from Canarium album. Planta Med. 55: 44-47. http://dx.doi.org/10.1055/s-2006-961822

Wang H, Song L, Tang H, He H, et al. (2010). [Main chemical components for clearing heat and relieving sore throat in different germplasm of Canarium album.]. Zhongguo Zhong Yao Za Zhi 35: 669-672.

Williams JG, Kubelik AR, Livak KJ, Rafalski JA, et al. (1990). DNA polymorphisms amplified by arbitrary primers are useful as genetic markers. Nucleic Acids Res. 18: 6531-6535.http://dx.doi.org/10.1093/nar/18.22.6531

Yang PK, Zhuang DH, Ma RJ, Zhao Q, et al. (2011). The ISSR analysis of genetic diversity of Canarium album L. germplasm resource in eastern Guangdong. Chin. Agric. Sci. Bull. 27: 86-92.

Zhan MM, Cheng ZZ, Su GC, Wang AY, et al. (2015). Genetic relationships analysis of olive cultivars grown in China. Genet. Mol. Res. 14: 5958-5969. http://dx.doi.org/10.4238/2015.June.1.13

Genetics and Molecular Research 15 (3): gmr.15038347 
Zhang C, Mei Z, Cheng J, He Y, et al. (2015). Development of SCAR markers based on improved RAPD amplification fragments and molecular cloning for authentication of herbal medicines Angelica sinensis, Angelica acutiloba and Levisticum officinale. Nat. Prod. Commun. 10: 1743-1747.

Zhang LL and Lin YM (2008). Tannins from Canarium album with potent antioxidant activity. J. Zhejiang Univ. Sci. B 9: 407-415. http://dx.doi.org/10.1631/jzus.B0820002

Genetics and Molecular Research 15 (3): gmr.15038347 